\title{
Modern lexicology of the French language within the framework of the concept of the digital economy
}

\author{
[La lexicologie courante de la langue française dans le cadre du concept \\ d'économie numérique]
}

\author{
Larisa E. Babushkina - Natalia V. Yudina - Olga A. Kalugina
}

DOI: 10.18355/XL.2021.14.04.08

\begin{abstract}
The purpose of the study is to describe the external structure of French lexicological terms with the identification of frame structures that are common for modern French lexicology and specific for a concept of "Digital Economy". The article presents the characteristics of the concept of "digital economy", carried out a frame analysis of the structure of the concept "digital economy". The scientific novelty of the research lies in the fact that for the first time on the empirical basis, the primary structure of the concept-term "digital economy = économie numérique" in French lexicology has been revealed. As a result, it is proved that the frame structure of this concept is atomic, hierarchical, and similar to the structure of other frames. The authors plan to clarify slots and sub-slots that were not originally included in the frame description. The theoretical significance of the research is associated with the possible use of its results in linguodidactics, terminology, and terminography. The practical value can be determined by the possible use of materials and research results in the practice of teaching French as a foreign language. The practical value lies in the frame analysis of the concept "digital economy" disclosed in the article, which can be used as the basis for elective disciplines in the field of the linguistic theory. The conclusions of this work can serve as a basis for compiling a database on the concept of "digital economy".
\end{abstract}

Key words: concept, digital economy, frame analysis, empirical material, frame structures, linguocultural aspect

\section{Résumé}

L'objectif de la recherche est de décrire l'apparence extérieure (forme) des termes lexicologiques français avec l'identification de structures de cadres communes à la lexicologie moderne de la langue française et spécifiques à un concept d'économie numérique. L'article présente les caractéristiques du concept d'économie numérique. Les auteurs ont réalisé une analyse de cadre de la structure du concept d'économie numérique. La nouveauté scientifique de la recherche est de se retrouver au fait que pour la première fois sur la base empirique de la recherche la structure primaire du concept "économie numérique" dans la lexicologie courante française a été révélée. En conséquence, il est prouvé que la structure de cadre de ce concept est atomique, hiérarchique et similaire à la structure des autres cadres. Les auteurs prévoient de clarifier les slots et les sous-slots qui n'étaient pas initialement inclus dans la description de la cadre. L'importance théorique de la recherche est associée à l'utilisation possible de ses résultats en linguistique, terminologie et terminographie. La valeur pratique peut être déterminée par l'utilisation possible de matériels et de résultats de recherche dans la pratique de l'enseignement du français langue étrangère. La valeur pratique réside dans l'analyse de cadre du concept "économie numérique" divulgué dans l'article, qui peut être utilisé comme base pour les disciplines électives dans le domaine de la théorie de la linguistique. Les conclusions de ce travail peuvent servir de base à la constitution d'une base de données sur le concept "économie numérique".

Mots-clés : concept, économie numérique, analyse de cadres, matériel empirique, structure de cadres, lexicologie courante de la langue française 


\section{L'introduction}

En raison du développement rapide de tous les types de communication interculturelle, l'étude des langues étrangères (et notamment de la langue française) revêt une importance particulière, car le degré d'efficacité de la communication professionnelle dépend de la qualité du vocabulaire utilisé. Depuis plusieurs décennies, la description de la terminologie professionnelle est un travail terminologique traditionnel (Boldyrev, 2016: 19), qui peut être réalisée à la fois par des spécialistes du domaine et par des linguistes ayant une formation en terminologie.

Cet article présente l'expérience de description de la terminologie lexicologique française courante. Une telle description peut faire partie du travail de rationalisation de la terminologie linguistique dans un domaine spécifique, en particulier "économie numérique".

La pertinence du sujet de recherche est de se retrouver au fait que l'économie numérique a un impact sur la sphère socio-culturelle et est l'une des sphères phares de l'activité humaine courante, ainsi qu'un moyen d'objectiver l'image linguistique du monde. La modélisation cognitive du concept "économie numérique" et le travail avec les cadres sont les plus pertinents dans le développement de la théorie de l'intelligence artificielle, qui permet de recréer des concepts culturels dans la conscience linguistique.

Il est à noter qu'à ce stade il n'y a ni un concept unique d'économie numérique, ni la structure de ce concept. La question de la possibilité d'unifier le concept d'économie numérique et de développer la structure de ce concept reste controversée et présente un intérêt important pour les théoriciens de la linguistique et les économistes pratiques.

$\mathrm{Au}$ cours de cette recherche, il est nécessaire de résoudre les tâches suivantes: d'une part, présenter les caractéristiques du concept d'économie numérique; d'autre part, présenter la structure primaire du concept d'économie numérique et la clarifier graphiquement, ce qui contribuera à refléter la diversité du fragment de l'image linguistique du monde dans lequel ce concept est présenté.

\section{La revue de la littérature}

La base théorique de la recherche est présentée par des travaux scientifiques d'auteurs nationaux (Likhachev, 1993; Bulygina et Shmelev, 1997; Stepanov, 1997; Arutyunova, 1999; Popova et Sternin, 2001; Karasik, 2004; Pimenova, Kondrateva 2011; Slyshkin, 2004; Matveeva, 2010, Zaitsev et Vasbieva, 2020 etc.), ainsi que des recherches d'auteurs internationaux (Gévaudan, 2015; Mel'cuk et Clas Polguère, 1995; Frérot, 2018; Temmerman, 2000; L'Homme, 2004; etc.) qui envisagent diverses approches de l'interprétation du terme "concept" et de sa structure.

Les approches de l'interprétation du terme "concept". Le concept est l'unité de base de l'image linguistique du monde. Un grand nombre d'ouvrages scientifiques sont consacrés à la question du "concept", à la fois concept à part entière et concepts spécifiques sur l'exemple de diverses cultures linguistiques. Dans le cadre de nos travaux, il n'est pas possible de présenter une analyse de toutes les études réalisées dans ce domaine. Tournonsnous uniquement vers les recherches qui peuvent servir de base théorique à cette étude.

Pour l'instant, en science linguistique nationale, il existe quelques approches de base pour l'interprétation d'un "concept" basées sur le jugement que le concept dénote le contenu d'un concept, il est un synonyme de sens.

La première approche (Karasik, 2004; Pimenova, Kondrateva 2011; etc.) est basée sur l'aspect culturel où le concept est identifié aux composantes culturelles et cognitives. Karasik (2004) croit que la conscience linguistique est basée sur l'expérience de l'individu y compris les composants ethniques, culturels, sociaux, individuels qui sont les concepts. Pimenova, Kondrateva (2011) définit le concept comme une idée d'un fragment du monde, une idée d'objets du monde externe et interne d'une personne. 
Selon la seconde approche (Bulygina et Shmelev, 1997; Arutyunova, 1999; etc.) les concepts sémiotiques correspondent à des noms (classificateurs) exprimant la relation entre la forme et la catégorie idéale.

Les adhérents de la troisième approche (Likhachev, 1993; Kubryakova, 2009; etc.) pensent que le concept est un conducteur entre le mot et la réalité. Ainsi, Likhachev (1993) définit un concept comme une expression algébrique de sens ; comme quelque chose formé à la jonction du sens du dictionnaire d'un mot et de l'expérience populaire.

D'une perspective européenne, la linguistique s'inscrit dans la tradition ancienne des fondements psychologiques de la linguistique. Cela explique, à côté de nouvelles conceptions telles que la théorie des scénarios ("frames") et de la théorie du prototype, le succès de ce mouvement basé sur l'idée de l'ancrage cognitif du langage. Après avoir fait état de ces données historiques, Paul Gévaudan (2015) consacre une section importante aux concepts fondamentaux de la linguistique cognitive, tels que les mécanismes associatifs, les scénarios, les catégories prototypiques, les concepts "incarnés", la subjectivité etc. La recherche de Paul Gévaudan (2015) est vouée aussi aux applications de la linguistique cognitive en lexicologie diachronique et synchronique, en morphologie et en grammaire. Ces applications laissent entrevoir dans quelle mesure le courant de la linguistique cognitive est devenu une théorie à part entière.

Les principes de base de la Théorie Générale de la Terminologie ont été repris dans de nombreux recherches internationales de terminologie (Frérot, 2018; Temmerman, 2000; L'Homme, 2004; etc.), l'accent étant mis sur ce que la terminologie étudie les concepts avant les termes, les concepts ont une place fixe attribuée dans le système, il y a un seul terme pour un seul concept et un seul concept pour un seul terme.

La structure conceptuelle. Il existe diverses études pour déterminer la structure du concept. Popova et Sternin (2001) représentent le modèle des champs du concept comme suit: noyau, couches de base se concentrant autour du noyau. Ils représentent des technologies d'analyse et de description de concepts (Popova et Sternin, 2001). La séquence est tracée: des couches moins abstraites aux couches plus abstraites (Popova et Sternin, 2001: 64).

Il y a une nouvelle direction en Russie, c'est la théorie de l'ethnopsycholinguistique, qui implique l'inclusion d'une composante émotionnelle-évaluative, et qui reflète la tension ethnique dans le modèle psychologique du concept. La tâche principale consiste à modéliser la structure de la conscience linguistique et à décrire son évolution, en tenant compte des spécificités ethnoculturelles, ce qui nécessite la formulation de tâches spéciales qui distinguent cette section des tâches générales de la psycholinguistique et de la linguistique cognitive, et le développement des méthodes de recherche en l'ethnopsycholinguistique (Shakhovsky, 2008).

Les recherches de Slyshkin (2004) sont consacrées à l'étude des caractéristiques du concept linguoculturel. Il a défini le concept linguoculturel comme une unité mentale complexe, incluant une composante culturelle, et qui a trouvé une réflexion verbale afin de satisfaire des besoins communicatifs. Les caractéristiques de base du concept linguoculturel, selon lui, comprennent la complexité de l'être, la mentalité, l'incarnation linguistique à trois niveaux (potentiel systémique, potentiel sujet, réalisations textuelles), la présence d'une intrazone et d'un extrazone (Slychkin, 2004).

Il existe différents types de concepts. La couche de base est une composante de tout type de concept, représentant une image sensorielle spécifique, complétée par des couches cognitives qui démontrent son développement et ses relations avec d'autres concepts. Les couches cognitives sont le résultat de la cognition. Popova et Sternin (2001) revendiquent trois types de modèles conceptuels à un niveau, à plusieurs niveaux et à segment (Popova et Sternin, 2001).

Matveeva (2010), analysant diverses approches du terme de "concept", conclut que la structure du concept devrait inclure une composante de valeur, puisque la base de la formation d'un concept est un phénomène de réalité et, par conséquent, un objet d'évaluation. Pour évaluer un objet, une personne a besoin de le "passer" à travers elle-même, ce processus

XLinguae, Volume 14 Issue 4, October 2021, ISSN 1337-8384, eISSN 2453-711X 
conduit à la formation d'un concept. Les composantes conceptuelles et figuratives sont également mises en évidence. La formation de la composante conceptuelle est facilitée par des faits concernant le réel ou l'imaginaire. Le composant figuratif comprend des représentations naïves qui sont intégrées dans la langue; formes internes de mots et d'images stables servant à exprimer le concept (Matveeva, 2010).

Selon Kononova (2012) la structure du concept linguoculturel se compose des éléments suivants: figuratif, associatif, conceptuel, valeur, étymologique, historique (Kononova, 2012).

Comme principaux facteurs cognitifs de la représentation du concept, la plupart des chercheurs désignés identifient la capacité d'interagir, ainsi que la capacité d'agir comme un espace mental et une zone mentale.

Les études de Boldyrev et Dubrovskaya (2016) présentent un intérêt théorique dans le cadre de ce recherche selon lequel dans le processus de connaissance par une personne de la réalité environnante, à la suite de divers types d'activité, une image conceptuelle du monde se forme dans la conscience d'un individu. Cependant, l'activité cognitive humaine dépend d'un certain nombre de facteurs socioculturels, qui déterminent en outre le système conceptuel d'un individu. Une typologie des contextes socioculturels est en train de se former à partir de l'expérience humaine dans une perspective historique, définie par différentes catégories telles que la nationalité, l'âge, le territoire, la profession, etc.

Sur la base de la distinction entre les aspects systémiques et fonctionnels du contexte socioculturel, les scientifiques distinguent les types de connaissances suivants et leurs unités (types de concepts). Le premier type de concepts comprend la totalité des connaissances humaines sur le monde, qui sont accumulées par toute l'humanité dans le processus d'évolution et par chaque individu dans le processus de sa vie. Ces concepts thématiques sont différents pour chaque personne, et sont classés selon les domaines conceptuels-thématiques. Le deuxième type de concepts est celui des concepts opérationnels basés sur des concepts thématiques et utilisés par une personne dans un discours spécifique (Boldyrev et Dubrovskaya, 2016: 32).

Boldyrev (2019) présente une classification des concepts basée sur la fonction réalisée, mettant en évidence les concepts représentatifs, sémiotiques et interprétatifs. Les concepts représentatifs reposent sur une vision du monde dans son ensemble. Il existe plusieurs niveaux: concepts de base (gestalts), superordonnés (abstraits) et subordonnés (concrets). Selon les moyens de représentation, les concepts peuvent être phonologiques, lexicaux, phraséologiques, onomasiologiques, dérivatifs, morphologiques, syntaxiques, superphrasaux, textuels, supertextes, discursifs, etc.

Les concepts sémiotiques ou les significations conceptuelles individuelles sont formés et transmis dans les processus de communication et sont verbaux et non verbaux.

Les concepts d'interprétation sont des unités de nouvelles connaissances basées sur une connaissance collective $\mathrm{du}$ monde au sein du système conceptuel individuel d'un individu. Le classement peut être le suivant: métadescriptif, y compris verbal (linguistique), métadiscursif et non verbal, et modus: sélectif (filtrage), classification, négatif, probant, approximateurs, suprasegmental (intonation, tonalité), éthique, esthétique, évaluatif (Boldyrev 2016).

Dans des études internationales sur la conceptualisation du monde dans le langage, il est noté que dans l'activité linguistique, la connaissance de tout format agit comme un contexte cognitif pour la formation et la compréhension des significations linguistiques.

La socioterminologie développée par François Gaudin (2003) place le concept en discours où, tout en se construisant, il subit toutes sortes de tensions et se modifie en fonction de variables sociales et historiques. C'est à cette époque que se développe également la Théorie communicative de Maria Teresa Cabré (2003) pour qui la connaissance spécialisée se matérialise par des formes linguistiques appelées unités de connaissance spécialisée regroupant tout à la fois des symboles, des phrases entières mais aussi des morphèmes dérivationnels, des unités terminologiques et les collocations. On citera également le modèle 
sociocognitif de Rita Temmerman (2000) dont les préoccupations portent sur la variation terminologique et la dimension diachronique des termes et l'approche lexico-sémantique de Marie-Claude L'Homme (2004) qui «aborde le terme comme une unité lexicale dont la particularité est d'avoir un sens qu'on peut associer à un domaine de la connaissance humaine» (L'Homme, 2004: 37).

Des fondements théoriques et méthodologiques de la terminologie sont bien exposés notamment dans l'article d'Aurélie Picton, Cécile Fabre et Didier Bourigault (2008). On retiendra notamment que le texte est le point de départ de l'analyse et de la ressource à construire, l'approche étant résolument descriptive (du texte vers le terme), et non plus normative. Le terme n'existe pas à priori, il est le résultat d'un travail d'analyse en fonction du corpus et de l'application. En fonction du corpus, car le terminologue recense et décrit les unités/structures lexicales présentes dans le corpus (intérêt pour l'ensemble des catégories grammaticales), et l'expert les valide; en fonction de l'application, car le terminologue recense les unités/structures lexicales en fonction de de leur utilité dans l'application visée.

Puisqu'un concept agit comme un modèle cognitif complexe et culturellement significatif, une combinaison de plusieurs méthodes différentes est nécessaire pour l'étudier. Nous pensons que le concept est important à étudier non seulement dans son interprétation cognitive, mais aussi dans son interprétation culturelle.

Premièrement, en explorant le concept "économie numérique", nous serons guidés par l'opinion que le concept représente une structure de cadre multidimensionnelle, qui, selon le concept généralement accepté de Minsky (1979), représente un réseau de nœuds et de connexions entre eux (Minsky, 1979: 26).

\section{Les matériaux et les méthodes}

Pour l'analyse de cadre du concept d'économie numérique, les auteurs de l'article utilisent les méthodes de recherche suivantes: méthode scientifique générale (formation de la base théorique de la recherche) ; méthode conceptuelle (mise en évidence des paramètres sémantiques du concept d'économie numérique) ; analyse du cadre (mise en œuvre de l'analyse du cadre de la structure du concept d'économie numérique).

\section{Les résultats}

\section{Le concept d'économie numérique comme le modèle cognitif à fixer et organiser des connaissances dans l'aspect linguoculturel}

L'analyse cognitive de la structure du cadre du concept d'économie numérique a pris en compte le fait que le cadre est une structure cognitive à plusieurs niveaux. Par conséquent, il est possible de clarifier les slots présentés avec une étude plus approfondie du concept d'économie numérique.

La structure du cadre du concept d'économie numérique est représentée par une pyramide ou le sommet est le concept de cadre "l'économie numérique" ; ci-dessous sont des sous-cadres, divisées en slots et terminaux remplis de composants variables.

Dans le cadre des slots sélectionnés, nous considérerons le concept d'économie numérique en tant que modèle cognitif pour fixer et organiser les connaissances sur une certaine situation stéréotypée sous-jacente aux définitions de "l'économie numérique" présentées dans le discours scientifique. La comparaison de la structure du cadre en français et en russe nous donnera l'occasion d'identifier les stéréotypes universels et nationaux spécifiques qui caractérisent les processus économiques de différents pays, l'attitude de la société à l'égard de ces processus.

L'attitude de la société vis-à-vis de la compréhension du concept d'économie numérique évolue au fil du temps en lien avec les processus de transformation numérique. Le secteur de l'économie numérique est un secteur à fort potentiel de développement économique et social. Comprendre ce concept est en pleine mutation, y compris toutes les

XLinguae, Volume 14 Issue 4, October 2021, ISSN 1337-8384, eISSN 2453-711X 
entreprises basées sur les TICes, quel que soit le secteur d'activité. Ainsi, la nécessité d'harmoniser la compréhension entre acteurs et de consolider les relations de collaboration a donné naissance à l'idée d'élaborer un lexique. Les compagnies de taxis en sont un bon exemple. Il y a 20 ans, dans l'esprit d'une personne, une société de taxis ne pouvait pas être associée au concept d'économie numérique. Aujourd'hui, en raison de l'émergence des services électroniques, tels que Yandex.Taxi, Uber, etc., on peut référer ce domaine à l'économie numérique. Le monde change, la compréhension de ce monde, l'attitude envers lui. Par conséquent, l'image linguistique du monde, l'appareil conceptuel d'un certain nombre de domaines, la conceptosphère, la structure de cadre des concepts subissent un changement. Le concept d'économie numérique ne fait pas exception. Le nombre d'articles scientifiques consacrés à ce sujet est limité.

L'économie numérique est plus qu'un sous-domaine de l'économie industrielle. Il ne s'agit pas seulement de revisiter des questions classiques de l'économie industrielle comme la fixation des prix en ligne, les stratégies de différenciation entre les offres physiques et en ligne, la régulation des marchés numériques ou l'effet de la publicité, mais aussi d'aborder de nouvelles questions de recherche comme l'économie des plateformes, des big data et de la vie privée (Einav et Levin, 2014). L'économie numérique aborde aussi des questions propres à d'autres champs de l'économie comme l'économie du travail (Autor, 2015; Agrawal et al., 2015) ou l'économie géographique (Sinai et Waldfogel, 2004). L'économie numérique est enfin porteuse d'un nouveau paradigme méthodologique lié à l'existence de traces et de données massives et variées qui peuvent être collectées sur Internet ou via des objets connectés. Les chercheurs peuvent aussi mettre en œuvre des expérimentations à grande échelle et observer des comportements en temps réel. Selon Edelman (2012), "pour les données collectées par les chercheurs, Internet ouvre des possibilités exceptionnelles à la fois en augmentant la quantité d'informations disponibles pour les chercheurs et en réduisant les coûts de collecte d'informations par les chercheurs". Mais cette "révolution des données" comporte des coûts d'entrée pour les chercheurs susceptibles de créer ou renforcer des fractures numériques entre les équipes de recherche, selon qu'elles ont ou non accès à ces données et les compétences pour les exploiter (Pénard et Rallet, 2014).

Les chercheurs en sciences humaines et sociales, et en premier lieu les économistes, ont un rôle important à jouer pour mieux comprendre ces transformations en cours, pour analyser et mesurer les effets socioéconomiques des technologies, services et usages numériques. Ces recherches de nature micro et macro-économique, théorique et empirique s'inscrivent dans un nouveau champ de la science économique, "l'économie numérique" (Bourreau et Pénard, 2016).

\section{La structure du concept-terme de l'économie numérique}

À la suite des scientifiques susmentionnés, nous pensons qu'il est conseillé de commencer l'analyse du concept en se référant aux définitions, car le concept est une idée généralisée d'un objet ou d'un phénomène. Pour cet analyse, nous avons sélectionné les revues des bases de données internationales scientométriques "Scopus", "Web of Science", la base de données bibliographique nationale de citation scientifique "Russian Citation Index", car ces sources contiennent du matériel scientifique (Introduction to Digital Economy, 2017; Kerimov et Antonova 2020; Nazarov, 2018; Maksaev et al, 2021; Panckhurst et Cougnon, 2019 , etc.) qui ont été étudiés, testés et contiennent des informations fiables et vérifiées.

La recherche de sources dans une base de données scientométrique était limitée aux catégories suivantes: année, type d'accès (libre accès), mot-clé (l'économie numérique), type de source (revue), branche de la connaissance (économie/économétrie/finance). Afin d'identifier la structure du concept-terme "économie numérique", nous avons également analysé la disponibilité d'un glossaire/dictionnaire pour ce concept sur Internet (Vocabulaire de l'économie et des finances, 2012, Vocabulaire des actifs numériques, 2021, etc.).

Ainsi, la nécessité d'harmoniser la compréhension entre acteurs et de consolider les relations de collaboration a donné naissance à l'idée d'élaborer un lexique. L'objectif 
poursuivi est de contribuer à l'émergence d'une compréhension commune des termes essentiels dans le domaine du numérique (Lexique de l'économie numérique et des postes, 2020).

Ce dictionnaire contient 305 unités présentées en français. Termes couramment utilisés avec le préfixe cyber-: cybermonnaie, cyberattaques, cybercriminalité, cyberdéfense, cyberespace, cyber menaces, cybersécurité, cybersquatting (cybersquattage), cyberstratégie, cybersurveillance, cyber-espionnage, cybercriminel, cybercriminalité (Lexique de l'économie numérique et des postes, 2020). Cette couche de vocabulaire représente environ $4 \% \mathrm{du}$ nombre total de termes contenus dans ce glossaire sur l'économie numérique.

Considéré comme un outil de communication et de culture des bonnes pratiques, le présent lexique permettra de faciliter une synergie d'actions de tous ceux qui souhaitent intervenir ou qui interviennent déjà dans le secteur de l'économie numérique et des postes.

La prétention de ce lexique n'est nullement de présenter un ensemble exhaustif de définitions arrêtées, scientifiquement stabilisées et devant être partagées par tous. Il faut plutôt comprendre les définitions données comme des notions dont les significations varient selon les secteurs d'activité, les contextes d'utilisation et les acteurs concernés.

Il existe des termes répandus qui se rapportent à la crypto-monnaie: Adresse bitcoin, Altcoin, Binance, Bitcoin, Bitcoin cash, Bloc orphelin, Cléprivée, Clépublique, Cloud mining, Cryptojacking, Cryptographie asymétrique, Cryptomonnaie, Euro numérique, Ethereum classic, Ether, EVM (machine virtuelleEthereum), Ferme de Minage, Hashrate, Internet computer, Lightning network, MakerDAO, Minageen Pool, Multisignal (multi-signatures), Node,Oracle (cryptomonnaie), ParrainageBinance, Phrase de recuperation, Pool de minage,Récompense de Bloc (Block Reward), Ripple,USD Coin,Wallet déterministe, etc. (Le dictionnaire de la cryptomonnaie).

Une attention particulière est également accordée aux systèmes facilitant la mise en œuvre de divers processus dans l'économie numérique dans divers domaines, notamment le commerce en ligne, les paiements, la gestion des achats en ligne; comptabilité, contrôle, planification et gestion de la production, des ressources matérielles, humaines et financières de l'entreprise, etc. Les secteurs de marché du e-commerce sont également inclus dans le vocabulaire de l'économie numérique: préchoix en ligne, marque en ligne, magazine en ligne, publipostage en ligne, salon professionnel en ligne, services en ligne, vendeur, -euse en ligne, distributeur en ligne, consommateur en ligne, formule en ligne, aide en ligne, approvisionnement en ligne, candidat en ligne, commerce en ligne, détaillant en ligne, administration en ligne, économie en ligne, entreprise en ligne, formation en ligne, galerie marchande en ligne, gestion des ressources humaines en ligne, impression en ligne, publipostage en ligne, librairie en ligne (Vocabulaire de l'économie et des finances, 2012).

Les listes terminologiques publiées au Journal officiel couvrent de nombreux domaines techniques dans les secteurs de pointe de l'information et de la communication (l'internet, l'informatique), des sciences (la chimie et l'ingénierie nucléaire, les sciences et les techniques spatiales), de l'industrie (l'automobile, le pétrole), de l'économie et des finances, de la santé, de l'agriculture, de la défense, des transports, de la culture, en fait l'ensemble des domaines de compétence des différents ministères. Tous les termes publiés au Journal officiel se trouvent sur le site FranceTerme (www.franceterme.culture.fr). Ce site est consacré aux termes publiés au Journal officiel de la République française par la Commission d'enrichissement de la langue française. Plus de 8500 termes pour nommer en français les réalités nouvelles et les innovations scientifiques et techniques.

Azema et Lenzen, (2014) présentent le lexique des termes du monde numérique. Dans l'éducation, le terme "numérique" fait référence à l'ensemble des outils et des technologies numériques utilisées à des fins éducatives (de l'ordinateur à l'utilisation des réseaux sociaux, en passant par les logiciels spécialisés ou l'utilisation de dispositifs mobiles). Décrivant l'essence des technologies numériques, les auteurs clarifient également des systèmes spéciaux qui aident à gérer tout cela dans son ensemble: Alphabétisation numérique, Humanités numériques, outils numériques, nouveaux contenus numériques, Identité 
numérique, représentations numériques, objet numérique, adresses numériques, appareils photo numériques, Dispositif numérique, Espace Numérique de Travail, ressources numériques, Fracture numérique.

Il est à noter que le dictionnaire français des Actifs Numériques contient une commission d'enrichissement (liste des termes, expressions et définitions acceptés) (Tableau 1):

Tableau 1

Tableau d'équivalence des termes étrangers

\begin{tabular}{|c|c|c|}
\hline Terme français (1) & Domaine/sous-domaine & $\begin{array}{l}\text { Équivalent étranger } \\
\text { (2) }\end{array}$ \\
\hline actif numérique & $\begin{array}{l}\text { Finance- } \\
\text { Informatique/Internet }\end{array}$ & digital asset \\
\hline $\begin{array}{l}\text { automate exécuteur de } \\
\text { clauses }\end{array}$ & $\begin{array}{l}\text { Assurance- } \\
\text { Informatique/Internet }\end{array}$ & smart contract \\
\hline cyberjeton, n.m., jeton, n.m. & $\begin{array}{l}\text { Finance- } \\
\text { Informatique/Internet }\end{array}$ & $\begin{array}{l}\text { coin, crypto asset, } \\
\text { token }\end{array}$ \\
\hline $\begin{array}{l}\text { dispositif d'enregistrement } \\
\text { électronique partagé (DEEP) }\end{array}$ & $\begin{array}{l}\text { Finance- } \\
\text { Informatique/Internet }\end{array}$ & $\begin{array}{l}\text { distributed ledger } \\
\text { technology (DLT) }\end{array}$ \\
\hline $\begin{array}{l}\text { gestion des } \\
\text { numériques }(\mathrm{GAN})\end{array}$ & $\begin{array}{l}\text { Finance- } \\
\text { Informatique/Internet }\end{array}$ & $\begin{array}{l}\text { digital asset } \\
\text { management } \\
\text { (DAM) }\end{array}$ \\
\hline jeton, n.m., cyberjeton, n.m. & $\begin{array}{l}\text { Finance- } \\
\text { Informatique/Internet }\end{array}$ & $\begin{array}{l}\text { coin, crypto asset, } \\
\text { token }\end{array}$ \\
\hline $\begin{array}{l}\text { offre au public de } \\
\text { cyberjetons, offre de jetons }\end{array}$ & $\begin{array}{l}\text { Finance- } \\
\text { Informatique/Internet }\end{array}$ & $\begin{array}{l}\text { initial coin offering } \\
\text { (ICO) }\end{array}$ \\
\hline registre partagé & $\begin{array}{l}\text { Finance- } \\
\text { Informatique/Internet }\end{array}$ & distributed ledger \\
\hline
\end{tabular}

(1) Les termes en caractères gras sont définis dans la partie I (Termes et définitions).

(2) Il s'agit d'équivalents anglais, sauf mention contraire.

Source:https://www.legifrance.gouv.fr/download/pdf?id=faoNbIDIFbjBr3IBpEk1O 3caoaKmU8eluFBKkf289nc=

La catégorie des technologies de l'information et de la communication (TIC) est représentée par un grand nombre d'unités. Par exemple, NTIC - Nouvelle technologie d'information et de communication est un ensemble d'outils issus des nouvelles technologies de l'information et de la communication comme la téléphonie mobile, le micro-ordinateur, les 
logiciels, les modems, Internet... (Abilways Digital, URL: https://www.abilwaysdigital.com/sur-mesure).

L'économie numérique est bien représentée par un véritable laboratoire d'innovation, un recueil d'expériences individuelles a été développé pour répondre aux enjeux de tous les publics en éducation. Prenons le terme "Massive Open Online Courses (MOOC)" comme exemple (Tableau 2).

Tableau 2

Le terme "Massive Open Online Courses (MOOC)" dans différentes sources

\begin{tabular}{|c|c|c|c|}
\hline terme d & finition & terme définition & source \\
\hline $\begin{array}{l}\text { MOOC } \\
\text { Open } \\
\text { Courses) }\end{array}$ & $\begin{array}{r}\text { (Massive } \\
\text { Online }\end{array}$ & $\begin{array}{l}\text { Type de formation à } \\
\text { distance, à laquelle un } \\
\text { grand nombre de } \\
\text { participants peut s'inscrire } \\
\text { (d'où la terminologie } \\
\text { «Ouvert et Massif»). Les } \\
\text { participants communiquent } \\
\text { via différents outils } \\
\text { numériques en ligne, en } \\
\text { particulier via des } \\
\text { plateformes spécialisées. }\end{array}$ & $\begin{array}{l}\text { https://www.mdenp.gov.bf/file } \\
\text { admin/user_upload/storages/do } \\
\text { cuments/Lexique_de_1_econom } \\
\text { ie_numerique_et_des_postes.p } \\
\text { df }\end{array}$ \\
\hline $\begin{array}{l}\text { MOOC } \\
\text { Open } \\
\text { Courses) }\end{array}$ & $\begin{array}{r}\text { (Massive } \\
\text { Online }\end{array}$ & Cours en ligne ouvert à tous & www.franceterme.culture.fr \\
\hline $\begin{array}{l}\text { MOOC } \\
\text { Open } \\
\text { Courses) }\end{array}$ & $\begin{array}{r}\text { (Massive } \\
\text { Online }\end{array}$ & $\begin{array}{l}\text { CLOM: Acronyme de } \\
\text { «Cours en Ligne Ouvert et } \\
\text { Massif» }\end{array}$ & $\begin{array}{l}\text { http://ww2.ac- } \\
\text { poitiers.fr/ecogest/sites/ecogest } \\
\text { /IMG/pdf/lexique_numerique.p } \\
\text { df }\end{array}$ \\
\hline $\begin{array}{l}\text { MOOC } \\
\text { Open } \\
\text { Courses) }\end{array}$ & $\begin{array}{r}\text { (Massive } \\
\text { Online }\end{array}$ & $\begin{array}{l}\text { Formation dispensée sur } \\
\text { Internet et accessible à tous. } \\
\text { (Recommandation } \\
\text { officielle: cours en ligne } \\
\text { ouvert à tous.) [Ce type de } \\
\text { cours offre à chacun la } \\
\text { possibilité d'évaluer ses } \\
\text { connaissances et peut } \\
\text { déboucher sur une } \\
\text { certification } \\
\text { payante).] (parfois }\end{array}$ & $\begin{array}{l}\text { https://www.larousse.fr/diction } \\
\text { naires/francais/MOOC/109108 } \\
87\end{array}$ \\
\hline $\begin{array}{l}\text { MOOC } \\
\text { Open } \\
\text { Courses) }\end{array}$ & $\begin{array}{r}\text { (Massive } \\
\text { Online }\end{array}$ & $\begin{array}{l}\text { Les cours pédagogiques } \\
\text { disponibles sur Internet et } \\
\text { de manière libre (gratuite). } \\
\text { L'échange communautaire } \\
\text { des connaissances est } \\
\text { valorisé. Le ITypa (Internet } \\
\text { Tout y est pour apprendre) } \\
\text { en est un exemple. }\end{array}$ & $\begin{array}{l}\text { https://www.abilways- } \\
\text { digital.com/sur-mesure }\end{array}$ \\
\hline
\end{tabular}

Le site Abilways Digital contient également un grand nombre de termes liés à l'économie numérique qui est exprimé verbalement par 77 moyens lexicaux (Abilways Digital, URL: https://www.abilways-digital.com/sur-mesure). Abilways est le groupe de formation professionnelle qui propose la plus large offre de formation dans le secteur du digital. Fort de son expertise et de son expérience, Abilways accompagne les entreprises et les 
organismes sur leurs problématiques stratégiques et opérationnelles. Abilways digital est une start-up interne propulsée par le groupe Abilways.

Cette ressource d'information présente les lexiques de la transformation digitale. Il est à noter que le glossaire contient des combinaisons terminologiques utilisant des néonomations avec le mot "digital". Cette unité est un anglicisme, le centre sémantique d'un grand nombre de néo-nominations analytiques. Un groupe lexico-sémantique est formé avec des relations sémantiques-paradigmatiques: le digital, la transformation digitale, l'informations digitales, la mue digitale, le Chief Digital Officer (ou CDO), la communication digitale, les digital natives, la nouvelle opération digitale, la sphère digitale, la publicité digitale, etc. (Abilways Digital, URL: https://www.abilways-digital.com/sur-mesure).

Sur la base de définitions qui donnent une détermination d'une unité lexicale, ainsi que d'une analyse du contenu des glossaires par domaine, le contenu minimum du concept de l'économie numérique: une activité qui s'appuie sur les technologies de l'information et de la communication et couvre divers processus. L'économie numérique est une activité réalisée grâce aux technologies de l'information et de la communication, couvrant divers processus. En analysant les définitions, nous avons identifié les nœuds sommets (de base) du cadre activité, technologies de l'information et de la communication, processus. Afin de déterminer les slots du cadre de l'économie numérique, nous avons analysé des fragments de glossaires. Graphiquement, la structure du cadre du concept de l'économie numérique est présentée comme suit (Figure 1):

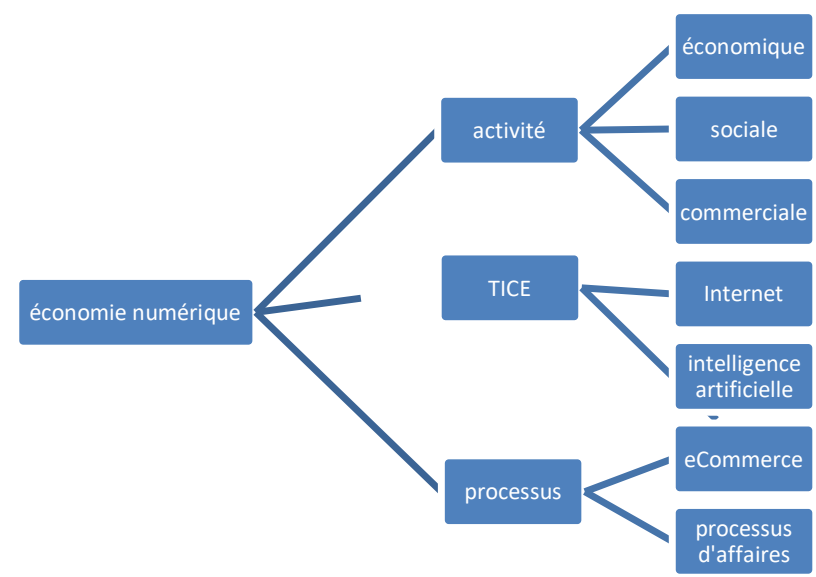

Figure 1. La structure du cadre du concept de l'économie numérique

\section{Discussion}

L'idée principale posée dès le premier paragraphe de cet article - qu'est-ce qui est le concept de l'économie numérique? - nous a emmenés dans un parcours de découvertes théoriques et pratiques. Le résultat de ce parcours est une meilleure compréhension des différentes significations du mot et de ce que peut apporter la lexicologie courante aux métiers de la traduction.

L'apprentissage des termes lexicologiques français avec l'identification de structures de cadres communes à la lexicologie moderne de la langue française et spécifiques à un concept d'économie numérique comporte un certain nombre de défis qui doivent être surmontés dans un avenir proche:

- les défis de nature personnelle: une faible auto-motivation initiale des étudiants en raison de la forme inhabituelle de l'enseignement à distance lui-même, énervement, attitude détendue au travail; 
- l'habituation au contrôle des enseignants, une faible auto-organisation des apprenants;

- le manque de contact émotionnel et psychologique avec les autres apprenants, le manque d'appartenance.

Merzouk (2021) a réalisé une enquête de terrain à travers laquelle les besoins des apprenants ont été identifiés, ainsi que les stratégies pédagogiques utilisées par les formateurs afin de savoir comment ces formateurs parviennent à répondre aux besoins spécifiques de leurs étudiants. (Merzouk, 2021).

L'objectif de la recherche de Baghana, Slobodova Novakova et Birova (2020) est de décrire l'importance des facteurs idéologiques, linguistiques, historiques et sociolinguistiques qui affectent le fonctionnement et la place de la langue française (Baghana, Slobodova Novakova et Birova, 2020).

L'économie numérique est bien représentée dans l'éducation en raison de la multiplication des MOOC. L'observation réflexive des activités pédagogiques des étudiants en formation aux langues étrangères, élaborées à partir de l'utilisation des MOOC, et l'analyse du contenu des MOOC destinés à l'enseignement professionnel des langues étrangères aux étudiants sont envisagées dans les recherches (Kandeel, 2020, etc.).

Les problèmes susmentionnés peuvent être surmontés à condition que l'utilisation de la lexicologie courante soit correctement organisée, l'auto-amélioration continue dans la maîtrise du vocabulaire professionnel lié au concept de l'économie numérique. Cela augmente la motivation des enseignants et des étudiants à utiliser activement les concepts de l'économie numérique dans l'enseignement et l'apprentissage des langues.

\section{Conclusion}

Par conséquent, le matériel empirique analysé nous a permis de construire la structure de cadre du concept de l'économie numérique et de confirmer le fait que cette structure est atomique, hiérarchique et similaire à la structure des autres cadres. Un cadre, agissant comme une structure cognitive, comprend un certain nombre de sous-cadres: activité, TIC, processus. Ils se répartissent en plusieurs créneaux interconnectés: économique, commercial, social, Internet, intelligence artificielle, e-commerce, business process. Les emplacements incluent des informations de base et sont des unités linguistiques stylistiquement neutres.

Lors de l'analyse cognitive initiale de la structure du cadre du concept de l'économie numérique, nous avons pris en compte le fait que ce travail servira de sujet de recherche future et les slots et sous-slots identifiés du concept de l'économie numérique seront clarifiés. En conclusion, nous notons que l'analyse du cadre présentée du concept de l'économie numérique est initiale et vise à caractériser, identifier les contours généraux, et est de nature préliminaire, nécessitant des tests supplémentaires sur un matériel plus étendu.

\section{Bibliographic references}

AGRAWAL, A. - HORTON, J. - LACETERA, N. - LYONS, E. 2015. Digitization and the Contract Labor Market: A Research Agenda. In A. Goldfarb, S. Greenstein, C. Tucker (eds.), Economic Analysis of the Digital Economy, Chicago, IL: The University of Chicago Press. Available online: http://john-joseph-horton.com/papers/digitization_and_contract_labor.pdf Avis et communications. Avis Divers. Commission d'enrichissement de la langue française. Vocabulaire des actifs numériques (liste de termes, expressions et définitions adoptés). Journal officiel de la république française. n. 13, Available online: https://www.legifrance.gouv.fr/download/pdf?id=faoNbIDIFbjBr3IBpEk1O3caoaKmU8eluF BKkf289nc=

ARUTYUNOVA, N.D. 1999. Language and man's world. 2nd edition, rev. M.: Languages of Russian culture. ISBN:5-7859-0027-0. 
AUTOR, D. 2015. Why Are There Still So Many Jobs? The History and Future of Workplace Automation. In: Journal of Economic Perspectives, vol. 29, pp.3-30. ISSN: 0895-3309.

AZEMA, G. - LENZEN, M. 2014. Le dico du numérique. Lexique des termes du monde numérique. Version 4.0. Available online: http://ww2.acpoitiers.fr/ecogest/sites/ecogest/IMG/pdf/lexique_numerique.pdf

BAGHANA, J. - SLOBODOVA NOVAKOVA, K. - BIROVA, J. 2020. The French Language in sub-Saharan Africa: Revisited. In: Research Result. Theoretical and Applied Linguistics, vol. 6, n. 1, pp. 54-64. ISSN: 2313-8912.

BOLDYREV, N.N. 2016. The typology of concepts and linguistic interpretation. New Russia: traditions and innovations in language and the science of language: Materials of reports and messages of the International Scientific Conference dedicated to the anniversary of the Honored Scientist of the Russian Federation, Doctor of Philology, Professor L. G. Babenko, Moscow-Yekaterinburg, / Editorial board: T. M. Voronina, M. V. Dudorova, B. Yu. Norman (Belarus), A. M. Plotnikova, H. Tommola (Finland). - Moscow-Yekaterinburg: Comics Factory LLC (imprint "Scientist Cabinet"), pp. 16-25. Available online: https://elar.urfu.ru/bitstream/10995/42885/1/nr_2016_02.pdf

BOLDYREV, N.N. - DUBROVSKAYA, O.G. 2016. Sociocultural context of the interpretation of the world and the formation of discourse. In: News of the Russian Academy of Sciences. A series of literature and language, vol. 75, n. 1, pp. 29-39. ISSN: 1605-7880.

BOLDYREV, N.N. 2019. Language and knowledge system. Cognitive theory of language. 2nd edition. Moscow: YASK Publishing House. 480 p. ISBN: 9785907117211.

BULYGINA, T.V. 1997. Linguistic conceptualization of the world (based on Russian grammar). Moscow: School "Languages of Russian Culture", 577 p. ISBN: 5-88766-051-1.

BOURREAU, M. - PENARD, T. 2016. Introduction. L'économie numérique en question. Revue d'Economie Industrielle, vol. 156, n. 4, pp. 11-15. Available online: https://journals.openedition.org/rei/6437

KESHELAVA, A.V. - BUDANOV, V.G. - RUMYANTSEV, V.YU. et al. 2017. Introduction to the "Digital" economy. "Digital." cons. I.A. Zimnenko. VNIIGeosystem, 28 p. Available online: http://spkurdyumov.ru/uploads/2017/07/vvedenie-v-cifrovuyuekonomiku-na-poroge-cifrovogo-budushhego.pdf

CABRÉ, M.-T. 2003. Theories of Terminology: Their description, prescription and explanation. In: Terminology, vol. 9, n. 2, pp. 163-199. Available online: https://www.researchgate.net/publication/233586525_Theories_of_terminology_Their_descri ption_prescription_and_explanation

GAUDIN, F. 2003. Socioterminologie. Une approche sociolinguistique de la terminologie, Bruxelles, De Boeck/Duculot.Available online: https://www.erudit.org/fr/revues/meta/2004v49-n2-meta770/009359ar.pdf

GÉVAUDAN, P. 2015. 24. La linguistique cognitive. In C. Polzin-Haumann \& W. Schweickard (Ed.), Manuel de linguistique française. Berlin, München, Boston: De Gruyter. pp. 585-617. Available online: https://doi.org/10.1515/9783110302219-026

EINAV, L. - LEVIN, J. 2014. Economics in the age of Big Data. In: Science, 346, n. 6210. Available online: https://web.stanford.edu/ leinav/pubs/Science2014.pdf

FRÉROT, C. 2018. Enseignement de la terminologie appliquée à une formation universitaire professionnalisante: illustration d'une collaboration avec l'Organisation Mondiale de la Propriété Intellectuelle. Myriades (ISSN: 2183-4989), Université du Minho (Braga, Portugal), (hal-02067277) Available online: http://cehum.ilch.uminho.pt/myriades/static/volumes/43.pdf.

KANDEEL, R. H. 2020. LMOOC for developing teaching and learning French as a foreign language (FFL) [Les LMOOC pour developper l'enseignement/ apprentissage du français langue etrangere (FLE)]. XLinguae. vol. 13 n. 1, pp. 24-36. ISSN: 1337-8384.

KARASIK, V.I. 2004. Language circle: personality, concepts, discourse. M.: Gnosis, ISBN: 9785733301433. 
KERIMOV, E.E. - ANTONOVA, N.L. 2020. Digital economy as a quality management tool. In: ModernScience, n. 6-1, pp. 97-102. ISSN: 2414-9918.

KONONOVA, I.V. 2012. The structure of the linguocultural concept: methods of identification and mechanisms of semantization. In: Pushkin Leningrad State University Journal, vol.7, n. 1, pp. 49-60. ISSN: 1818-6653.

KUBRYAKOVA, E.S. 2009. About concepts captured by the sign. In: Studia Linguistica (St. Petersburg), n. 18. pp. 69-75. ISBN: 978-5-904030-76-6.

Larousse. Available online: https://www.larousse.fr/dictionnaires/francais/MOOC/10910887

Lexique de l'economie numerique et des postes. Ministere du developpement de l'economie numerique et des postes, 2020. Available online: https://www.mdenp.gov.bf/fileadmin/user_upload/storages/documents/Lexique_de_1_econom ie_numerique_et_des_postes.pdf

Le dictionnaire de la cryptomonnaie. Available online: https://www.journaldunet.fr/patrimoine/guide-des-finances-personnelles/le-dictionnaire-de-lacryptomonnaie/

L'HOMME, M.-C. 2004. La Terminologie: principes et techniques. Presses de l'Université de Montréal. ISBN: 9782760619494.

LIKHACHEV, D.S. 1993. The conceptual framework of the Russian language. Available online: https://www.lihachev.ru/lihachev/bibliografiya/4946/

MAKSAEV, A.A. - VASBIEVA, D.G. - SHERBAKOVA, O.Y. - MIRZOEVA, F.R. KRALIK, R. 2021. Education at a cooperative university in the digital economy. In: Studies in Systems, Decision and Control, vol. 316, pp. 33-42. ISSN: 2198-4182, ISBN 978-3-03057830-5.

MATVEEVA, D.S. 2010. Concept as a unit of consciousness. In: Bulletin of Volzhsky University after V.N. Tatischev, n. 6, pp. 94-99. ISSN 2076-7919.

MERZOUK, S. 2021. D'un enseignement général du FLE vers des enseignements spécifiques à l'université de Sétif: une transition sans formation des formateurs. In search of the LSP teacher's competencies [À la recherche des compétences des enseignants de LS]. Bern, Switzerland. Available

online:

https://www.peterlang.com/view/9783631823200/html/ch21.xhtml

MEL'CUK, I.A. - CLAS, A. - POLGUÈRE, A. 1995. Introduction à la lexicologie explicative et combinatoire. Editions Duculot. 256 p. ISBN: 2-8011-1106-6.

MINSKY, M. 1979. Frames for knowledge representation / ed. F.M. Kulakov. Moscow: Energy, 151p. Available online: http://hegelnet.org/intsys/literature/minsky-frames.pdf

NAZAROV, D.M. 2018. The digital economy as a result of information revolutions. In: Bulletin of the St. Petersburg State University of Economics, vol. 5 (113), pp. 12-24. ISSN 2311-3464.

POPOVA, Z.D. - STERNIN, I.A. 2001. Essays on Cognitive Linguistics. Voronezh: Istoki, 191 p. ISBN: Po5-88242-189-6.

PIMENOVA, M.V. - KONDRATEVA, O.N. 2011. Conceptual studies. Introduction. Flinta, 210p. ISBN: 978-5-9765-1058-6.

PANCKHURST, R. - COUGNON, L. 2019. Youth digital practices: Results from belgian and french projects. TechTrends, vol. 63, n. 6, pp. 741-750. Available online: https://link.springer.com/article/10.1007/s11528-019-00417-y

PICTON, A. - FABRE, C. - BOURIGAULT, D. 2008. Méthodes linguistiques pour l'expansion de requêtes. Une expérience basée sur l'utilisation du voisinage distributionnel. Revue française de linguistique appliquée, vol. 8, pp. 83-95. ISSN: 1386-1204.

PÉNARD, T. - RALLET, A. 2014. De l'économie des réseaux aux services en réseaux: nouveau paradigme, nouvelles orientations. Réseaux, pp. 184-185, pp.71-93. ISBN: 9782707179043.

SINAI, T. - WALDFOGEL, J. 2004. Geography and the Internet: Is the Internet a Substitute or a Complement for Cities? Journal of Urban Economics, vol. 56, pp.1-24. Available online: https://reader.elsevier.com/reader/sd/pii/S0094119004000439?token=9407A998A73DBA15D

XLinguae, Volume 14 Issue 4, October 2021, ISSN 1337-8384, eISSN 2453-711X 
D214768B6BA30C676D6AF1EA8929BF642E0CF36B5F79E6F24D8D0F06E4859A7EFDA 57BB8B0FCC9F\&originRegion=eu-west-1\&originCreation=20210919133658

SLYSHKIN, G.G. 2004. Linguocultural concepts and meta-concepts: thesis abstract for the degree of Doctor of Philology. Volgograd, 39 p. Available online: https://newdisser.ru/_avtoreferats/01003299039.pdf

STEPANOV, YU.S. 1997. Constants. Dictionary of Russian culture. Research experience. Moscow: School of the Language of Russian Culture, 824 p. ISBN: 5-88766-057-0.

TEMMERMAN, R. 2000. Towards New Ways of Terminology Description. The sociocognitive approach. Amsterdam/Philadelphie. John Benjamins. ISBN: 9789027223265.

SHAKHOVSKY, V.I. 2008. Book review: Pishchalnikova V.A. History and theory of psycholinguistics: a course of lectures. Part 2. Ethnopsycholinguistics. Available online: http://grani.vspu.ru/files/publics/18_pub.pdf

VOCABULAIRE DE L'ÉCONOMIE ET DES FINANCES. ENRICHISSEMENT DE LA LANGUE FRANÇAISE. 2012. ISSN imprimé: 1961-9790 - ISSN en ligne: 1961-9804. Available online: https://www.academie-francaise.fr/sites/academiefrancaise.fr/files/economie_finances_2012.pdf

ZAITSEV, A.A. - VASBIEVA, D.G. 2020. Peculiarities of using demonstrative pronoun ça in the modern French language, Les particularites de l'emploi du pronom demonstratif ça en Français contemporain. In: XLinguae, vol.13, n. 1, pp. 194-203. ISSN 1337-8384.

Words: 6619

Characters: 49153 (27,31 standard pages)

Assoc. Prof. Babushkina Larisa Evgenievna, $\mathrm{PhD}$

Institute of Economics and Management of Agroindustrial Complex

Foreign and Russian Languages Departement

Russian State Agrarian University - Moscow Timiryazev Agricultural Academy

Timiryazevskaya st., 49

127550 Moscow

Russia

lb_77@list.ru

Professor Yudina Natalia Vladimirovna, Doctor

Department of Foreign Languages and Intercultural Communication

Financial University under the Government of the Russian Federation

Leningradskiy prospekt, 49

12593 Moscow

Russia

nvyudina@fa.ru

Assoc. Prof. Kalugina O.A.

Department of English Language for Professional Communication

Financial University under the Government of the Russian Federation

Leningradskiy prospekt, 49

12593 Moscow

Russia

kaluginaruc@mail.ru 